\title{
Academic Community's Efforts to Guide the Fight Against Coronavirus Disease 2019 (COVID-19) Epidemic in Korea
}

\author{
Sung-il Cho \\ Department of Preventive Medicine, Graduate School of Public Health, Seoul National University, Seoul, Korea
}

With the rapid spread of coronavirus disease 2019 (COVID-19), the Korean government raised the epidemic response stage to the highest "red alert" level on February 23, 2020, and has been making great efforts to mitigate the effects of community transmission. Such measures have been taken to follow the recommendations given on the day before by the joint committee of academic and professional organizations to respond to the COVID-19 epidemic in Korea. In the recommendations, the committee called for a transition from a containment strategy in response to the limited COVID-19 transmission to a mitigation strategy in response to community spread or an impact minimization strategy.

While the containment strategy focused on contact investigation for confirmed patients and prevention of further spread, the mitigation strategy focuses on reducing damage in the context of continued community transmission. As containment and mitigation are not mutually exclusive, it is necessary to continue containment efforts. Even if the blockade is incomplete, the benefit of slowing the spread to communities remains substantial. The main limitation of the containment strategy is asymptomatic transmission, as COVID-19 could be infectious even when symptoms are mild or absent. Such cases have been uncommon during the early epidemic stage in Korea, and it has been possible to find links of transmission for the majority of early cases. However, limits of the containment strategy emerged

\section{Corresponding author: Sung-il Cho, MD, ScD}

Department of Preventive Medicine, Graduate School of Public Health, Seoul National University, 1 Gwanak-ro, Gwanak-gu, Seoul 08826,

Korea

E-mail: scho@snu.ac.kr

This is an Open Access article distributed under the terms of the Creative Commons Attribution Non-Commercial License (https://creativecommons.org/licenses/bync/4.0/) which permits unrestricted non-commercial use, distribution, and reproduction in any medium, provided the original work is properly cited. as cases with unknown routes of infection increased. The pathways of infection are difficult to determine because of asymptomatic transmission. Further, determining the pathways is difficult in cases where epidemiological investigation was unable to assess information on close contacts of the confirmed cases, either by non-disclosure of information or the surge of demand overflowing the capacity of epidemiologic investigators. In this context, social distancing must be particularly emphasized. The situation is evolving rapidly, and continued analysis of the data collected is crucial. Academic organizations are playing important roles to provide guidance to the government authorities and to the public during this epidemic period. Here, we attach as supplements the recommendations jointly provided by academic organizations so far in Korea for an interim review. The original Korean versions have been already posted in the homepages of the participating academic organizations, including the Korean Society for Preventive Medicine and the Korean Society of Epidemiology. The attached English versions (Supplemental Materials 1-6) are provided by the translation team serving jointly for these two societies, and may not be the translation officially approved by all the participating organizations.

\section{SUPPLEMENTAL MATERIALS}

Supplemental materials are available at https://doi.org/10. 3961/jpmph.20.084.

\section{CONFLICT OF INTEREST}

The authors have no conflicts of interest associated with the material presented in this paper. 


\section{ACKNOWLEDGEMENTS}

We thank SuJin Kang, Jihyun Moon, and Heewon Kang for providing the English translations for the joint statements.

\section{ORCID}

Sung-il Cho https://orcid.org/0000-0003-4085-1494 